STRUCTURAL BIOLOGY COMMUNICATIONS

ISSN 2053-230X

Received 8 June 2018

Accepted 9 July 2018

Edited by R. L. Stanfield, The Scripps Research Institute, USA

Keywords: antibodies; NIST reference antibody RM8671; conformation; crystal structure; Fc; human; immune; X-ray.

PDB reference: Fc fragment of human IgG1 antibody from NISTmAb, 5vgp

Supporting information: this article has supporting information at journals.iucr.org/f

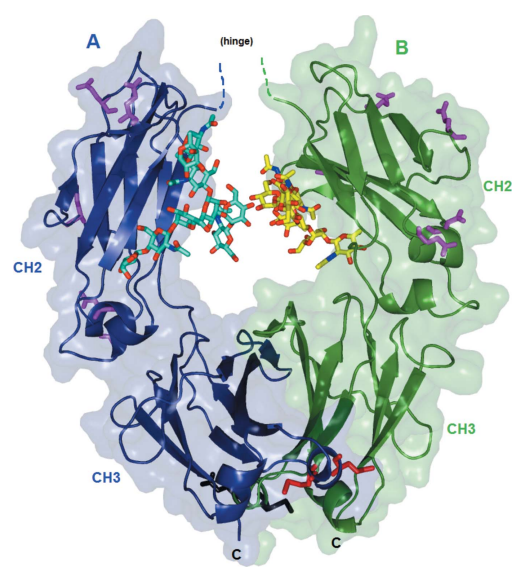

OPEN $\odot$ ACCESS

\section{Structure of the Fc fragment of the NIST reference antibody RM8671}

\author{
D. Travis Gallagher, ${ }^{a *}$ Connor V. Galvin ${ }^{b}$ and Ioannis Karageorgos ${ }^{a}$
}

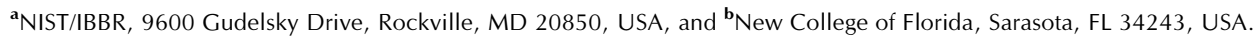
*Correspondence e-mail: gallagher@ibbr.umd.edu

As the link between antigen binding and immune activation, the antibody $\mathrm{Fc}$ region has received extensive structural study. In this report, the structure of the Fc fragment of the NIST IgG1 mAb (reference material 8671) is described at $2.1 \AA$ resolution in space group $P 2_{1} 2_{1} 2_{1}$, with approximate unit-cell parameters $a=50, b=80, c=138 \AA$. Prior Fc structures with a wide variety of modifications are also surveyed, focusing on those in the same crystal form. To facilitate the analysis of conformations, a reference frame and a two-parameter metric are proposed, considering the $\mathrm{CH} 2$ domains as mobile with respect to a fixed dimeric $\mathrm{CH} 3$ core. Over several human $\mathrm{Fc}$ structures, a significant variation in $\mathrm{Fc}$ elbow conformations is observed, which may serve to facilitate the regulation of Fc effector signaling.

\section{Introduction}

The Fc (fragment crystallizing) of the $\operatorname{IgG}$ antibody was named by Rodney Porter, who found that it crystallized unexpectedly in his refrigerator (Porter, 1959). Using rabbit proteins, he was among the first to analyze the isolated Fab and Fc fragments; this work paved the way for molecular immunology and led to his 1972 Nobel Prize, which was shared with Gerald Edelman. The antibody Fc translates pathogen recognition into immune activation, but the regulation of this crucial signal involves a delicate balance dependent on structural details (Caaveiro et al., 2015). The binding of Fc to various types of $\mathrm{FcR}$ cellular receptors is crucial and is sensitive to variations in the glycan that is attached to the conserved Asn300 (the actual sequence number varies owing to differing CDR lengths, and many structures call this residue Asn297). Other key binding partners include C1q (Schneider \& Zacharias, 2012), which initiates the complement reaction, and neonatal FcRn (Oganesyan et al., 2014), which facilitates the transport of maternal antibodies into newborns.

The immune system functions by deploying a wide repertoire of specific molecular shapes, so that structural knowledge of antibodies has become a key resource in understanding immunity and in the engineering of immune-inspired molecular therapies (Kennedy et al., 2018). The Protein Data Bank (Berman et al., 2000) contains over 100 structures of human IgG Fc crystallized with variations in the glycan, in the protein sequence and in the crystallization conditions, and in various complexes (see Supplementary Table S1 for a summary of precedent Fc structures). Complexes with $\mathrm{FcR}$ are especially informative and include most of the known FcR subtypes. The set of uncomplexed human Fc structures includes one common recurring crystal form in space group $P 2_{1} 2_{1} 2_{1}$, with approximate unit-cell parameters $a=50, b=80, c=138 \AA$. This common crystal includes about 40 structures, but for 
Table 1

Source and sequence.

\begin{tabular}{ll}
\hline Source organism & Homo sapiens \\
Expression host & HEK293F cells \\
Complete amino-acid sequence & TCPPCPAPELLGGPSVFLFPPKPKDTLMIS \\
of the construct produced & RTPEVTCVVVDVSHEDPEVKFNWYVDGV \\
& EVHNAKTKPREEQYNSTYRVVSVLTVLH \\
QDWLNGKEYKCKVSNKALPAPIEKTISK \\
AKGQPREPQVYTLPPSREEMTKNQVSLT \\
CLVKGFYPSDIAVEWESNGQPENNYKTT \\
PPVLDSDGSFFLYSKLTVDKSRWQQGNV \\
FSCSVMHEALHNHYTQKSLSLSPGK \\
\hline
\end{tabular}

wild-type sequences its resolution is limited to about $2 \AA$. The highest resolution structures in this crystal form are at about $1.7 \AA$ and contain multiple mutations. The highest resolution Fc structures in general are complexes such as PDB entry $116 \mathrm{x}$ $(1.65 \AA)$ and stabilized mutants such as PDB entry 5jih $(1.7 \AA)$. Supplementary Table S1 gives information on 16 representative precedent Fc structures.

In order to support the development of antibody-based medicines, the National Institute of Standards and Technology (NIST) has released an extensively characterized IgG1 mAb, called reference material 8671 or NISTmAb. To provide a scientific description of this material and to facilitate its application, we report the crystal structure of its Fc fragment. This is now the highest resolution structure available with its specific sequence in the common orthorhombic form. We compare it with other high-resolution structures, emphasizing the variations in overall conformation, and develop a reference framework based on two geometric parameters to facilitate conformational analysis.

\section{Materials and methods}

\subsection{Macromolecule production}

The crystallized Fc was prepared from a starting antibody that is a humanized $\mathrm{IgGk} 1 \mathrm{mAb}$ produced recombinantly in HEK293F cells and is available as NIST reference material 8671 (Schiel et al., 2015). The Fc fragment was prepared from NISTmAb by papain cleavage as described in Karageorgos et al. (2017). After cleavage, the digest was applied onto a $5 \mathrm{ml}$ protein A agarose column (Pierce Nab column, Thermo Scientific, Waltham, Massachusetts, USA) that had been equilibrated with $25 \mathrm{ml}$ PBS and was incubated at room temperature for $20 \mathrm{~min}$ with agitation to capture the Fc and potential undigested NISTmAb. Fc and undigested mAb were eluted using the elution buffer from the Pierce Fab Preparation kit (Model 44985, Thermo Scientific) and Fc was separated from undigested mAb using a $100 \mathrm{kDa}$ cutoff Amicon Ultra centrifugal filter (Sigma-Aldrich, St Louis, Missouri, USA) followed by preparative gel-filtration (GF) chromatography (Supplementary Fig. S1). Prior to GF chromatography, the material, both nonreduced and BME-reduced, was analyzed by gel electrophoresis (Supplementary Fig. S2). After GF, the collected Fc peak was dialyzed into $20 \mathrm{mM}$ L-histidine buffer $\mathrm{pH} 6.0$ and was concentrated to $25 \mathrm{mg} \mathrm{ml}^{-1}$ for crystal screening. Macromolecule-production information is given in Table 1.

\subsection{Crystallization, data collection and structure determination}

Initial screening by vapor diffusion in sitting drops at $24^{\circ} \mathrm{C}$ produced crystals in ten out of 96 conditions. The conditions that gave the largest crystals were refined and optimized to reservoir conditions consisting of $40 \mathrm{~m} M \mathrm{CaCl}_{2}, 17 \%(w / v)$ PEG 8000, $100 \mathrm{~m} M$ sodium HEPES pH 7.0. Crystal growth was highly sensitive to the calcium concentration. Strontium appeared to substitute for calcium, but subsequent analysis failed to reveal any bound strontium site, suggesting that the role of the divalent cation in crystal growth is transient. Subsequent analysis of crystallization conditions in $38 \mathrm{Fc}$ structures of the same crystal form in the PDB (with various mutations and glycoforms etc.) revealed that over $90 \%$ had a $\mathrm{pH}$ of between 5.8 and 7.2 and used some form of polyethylene glycol, but they were ionically diverse and only two included divalent cations. A crystal of dimensions $300 \times 100 \times$ $50 \mu \mathrm{m}$ was dunked for $2 \mathrm{~s}$ into $15 \%(w / v)$ glycerol and $85 \%$ reservoir solution and then cryocooled in liquid nitrogen and kept at $-100^{\circ} \mathrm{C}$ for data collection. Diffraction data were collected to $2.1 \AA$ resolution on beamline 19-ID of the Advanced Photon Source (APS) at Argonne National Laboratory using an ADSC Q315r detector. Data in space group No. 19 were processed using $H K L-3000$ (Minor et al., 2006). The previously observed unit cell implied that the crystal was isomorphous to several precedent Fc structures. Refinement began with a model derived from the precedent structure with PDB code 3do3 (B. C. Braden, unpublished work), from which the glycans and hinge-proximal loops had been removed. Refinement used REFMAC in the CCP4 suite (Winn et al., 2011) and proceded in stages, gradually adding the missing parts and waters. In addition to general structure validation by the PDB, the glycans were validated by $p d b$-care at glycosciences.de (Emsley et al., 2015).

\subsection{Structure analysis}

PyMOL (DeLano, 2002) was used to generate molecular graphics as well as for structure alignment and r.m.s.d. calculations. PISA (Krissinel \& Henrick, 2007) was used for the calculation of buried surface areas. Statistics for the deposited structure (PDB entry 5vgp) are given in Tables 2 and 3. A reference frame for conformational analysis was developed beginning from a Cartesian origin defined at the center of gravity of the $\mathrm{CH} 3-\mathrm{CH} 3$ dimer and a dyad passing vertically through this point (see Fig. 1a). In some Fc structures this dyad is perfect (crystallographic), but in most it is only a local dyad and the two chains differ, giving a sample of conformational variation. The reference frame is then further defined by a horizontal plane through the centers of the two tightly associated $\mathrm{CH} 3$ domains. To avoid confusion with crystal axes, we label the reference axes $p, q$ and $r$, with $r$ as the vertical dyad. The centers of the two $\mathrm{CH} 2$ domains can then be plotted with respect to the dimeric $\mathrm{CH} 3$ core, and various structures 
with different elbow conformations can be superposed by their relatively rigid $\mathrm{CH} 3$ domains to observe the variation in their $\mathrm{CH} 2$ loci (see Fig. $1 b$ ). Although in principle each $\mathrm{CH} 2$ centroid has three degrees of freedom, the elbow appears to function as a ball-and-socket joint (Teplyakov et al., 2013) so that there are two main degrees of freedom, and the small vertical variation can be neglected. Using this system, Fig. 1(c) shows that a sampling of Fc structures, within the space group and beyond, shows a range of different conformations.

\section{Results and discussion}

\subsection{Structure of NISTmAb Fc and allotypic variation}

The deposited structure includes all protein atoms from the hinge double glycine (residues 239 and 240) to Ser447 in both chains (Fig. 2). The hinge residues 228-238 are disordered. Although excluded from the refined structure, weak electron density was observed for the entire hinge, including both disulfides (data not shown); the space between adjacent molecules appears to be just right to accommodate the hinge, and this may help to explain the common occurrence of this crystal form among many variations in the $\mathrm{Fc}$ that preserve the IgG1 hinge. The crystals gave significantly better diffraction from the $A$ chain relative to the $B$ chain (the mean isotropic $B$ values are 47 and $66 \AA^{2}$, respectively). The glycans include all atoms consistent with the biophysically characterized principal glycoform $\mathrm{G} 1 \mathrm{~F} / \mathrm{G} 0 \mathrm{~F}$ (i.e. the $A$-chain glycan has nine sugar groups including one fucose and one terminal galactose, while the $B$ chain has only eight, lacking galactose). The two glycans
Table 2

Data collection and processing.

\begin{tabular}{ll}
\hline Diffraction source & Beamline 19-ID, APS \\
Wavelength $(\AA)$ & 0.97934 \\
Temperature $(\mathrm{K})$ & 100 \\
Detector & PILATUS 6M \\
Crystal-to-detector distance $(\mathrm{mm})$ & 290 \\
Rotation range per image $\left({ }^{\circ}\right)$ & 0.8 \\
Total rotation range $\left({ }^{\circ}\right)$ & 180 \\
Exposure time per image (s) & 2 \\
Space group & $P 2_{1} 2_{1} 2_{1}$ \\
$a, b, c(\AA)$ & $49.91,79.96,138.35$ \\
$\alpha, \beta, \gamma\left({ }^{\circ}\right)$ & $90,90,90$ \\
Resolution range $(\AA)$ & $30.0-2.1(2.17-2.13)$ \\
Total No. of reflections & 207234 \\
No. of unique reflections & 30746 \\
Completeness $(\%)$ & $94.4(71.0)$ \\
Multiplicity & $6.7(3.3)$ \\
$\langle I / \sigma(I)\rangle$ & $7.2(2.6)$ \\
$R_{\text {r.i.m. }}^{\dagger}$ & 0.106 \\
Overall $B$ factor from Wilson plot $\left(\AA^{2}\right)$ & 40.032 \\
\hline
\end{tabular}

$\dagger$ Estimated $R_{\text {r.i.m. }}=R_{\text {merge }}[N /(N-1)]^{1 / 2}$, where $N$ is the data multiplicity.

interact through one hydrogen bond between mannose hydroxyls across the molecular dyad (Fig. 3). Other glycan interactions include several weak hydrogen bonds to the protein.

Most human Fcs with known crystal structures belong to one of three allotypes, correponding to polymorphisms that appear as sequence variations at seven specific sites, as shown in Fig. 2. In the structure with PDB code 5vgp these sites are Glu275, Glu286, Glu297, Asp315, Asn318, Glu359 and Met361. The first five of these are in $\mathrm{CH} 2$ and vary as a group, appearing as E275Q, E286Q, E297Q, D315N and N318D in

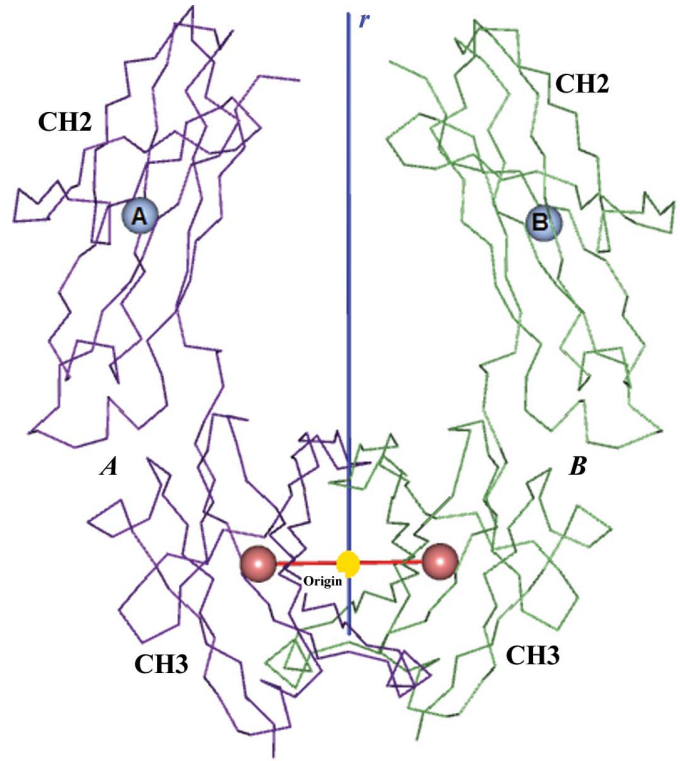

(a)

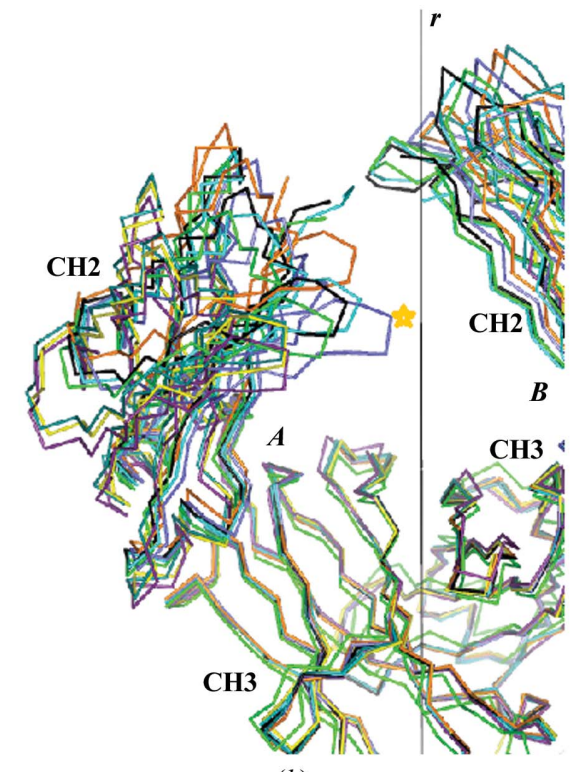

(b)

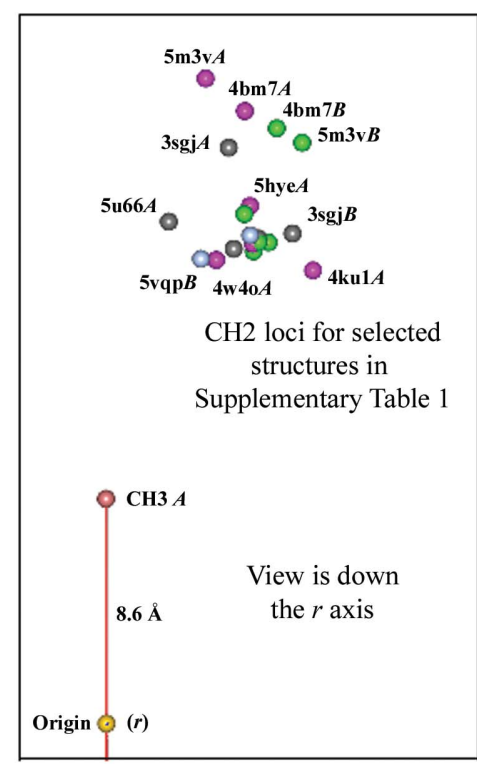

(c)

Figure 1

Geometric reference frame for Fc conformational analysis. (a) shows the least-squares dyad relating the tightly associated and structurally conserved $\mathrm{CH} 3$ domains. The centroids of the four domains are shown as spheres. Using this dyad as a vertical axis and the $\mathrm{CH} 3$ domain centroids to define the horizontal axes of a Cartesian frame, $\mathrm{CH} 2$ loci in different structures can be compared (after superposing their $\mathrm{CH} 3$ domains). (b) shows a superposition of seven Fc structures from the orthorhombic crystal form, showing that the $\mathrm{CH} 2$ domains have a wide range of positional variation. The star indicates the Asn residue to which the glycans attach. This point varies by up to $1.2 \mathrm{~nm}$ among the structures, suggesting that the glycans explore a large conformational space. $(c)$ shows the same data using the proposed reference frame, and includes two additional Fc structures: the complexes with PDB codes 3 sgj and 5 u66. 
the precedent structure PDB entry $1 \mathrm{~h} 3 \mathrm{t}$ and a few others. The last two of the seven variable sites are in $\mathrm{CH} 3$, and occur as E359D and M361L in PDB entries 4w4n, 5jii and several others. Allotypic variation in human populations is useful for identity testing but has unknown functional implications, and for therapeutic antibodies creates an unknown risk of immunogenicity (Vidarsson et al., 2014). Most of these sites are on the surface and most are involved (within $4 \AA$ ) in crystal-packing interactions so that they may also influence crystallization and resolution.

\subsection{Symmetry and packing}

Although the $\mathrm{Fc}$ is chemically symmetric or very nearly so, most $\mathrm{Fc}$ crystal structures are asymmetric. In the common orthorhombic crystal form in the present study, the asymmetric unit contains a complete Fc molecule and the two chains differ (Fig. 4). The differences are greatest in the $\mathrm{CH} 2$ domains, and in most structures one subunit is better ordered (with lower temperature factors) than the other. The better ordered (lower average $B$ ) subunit is generally the one that corresponds to the $A$ chain in PDB entry 5vgp, which is the one that is further from the $\mathrm{CH} 3$-based molecular dyad (Fig. 4a). This is consistent with the fact that the $A$-to- $A$ crystal packing contact is significantly larger than the $B$-to- $B$ packing contact (Supplementary Fig. S5).

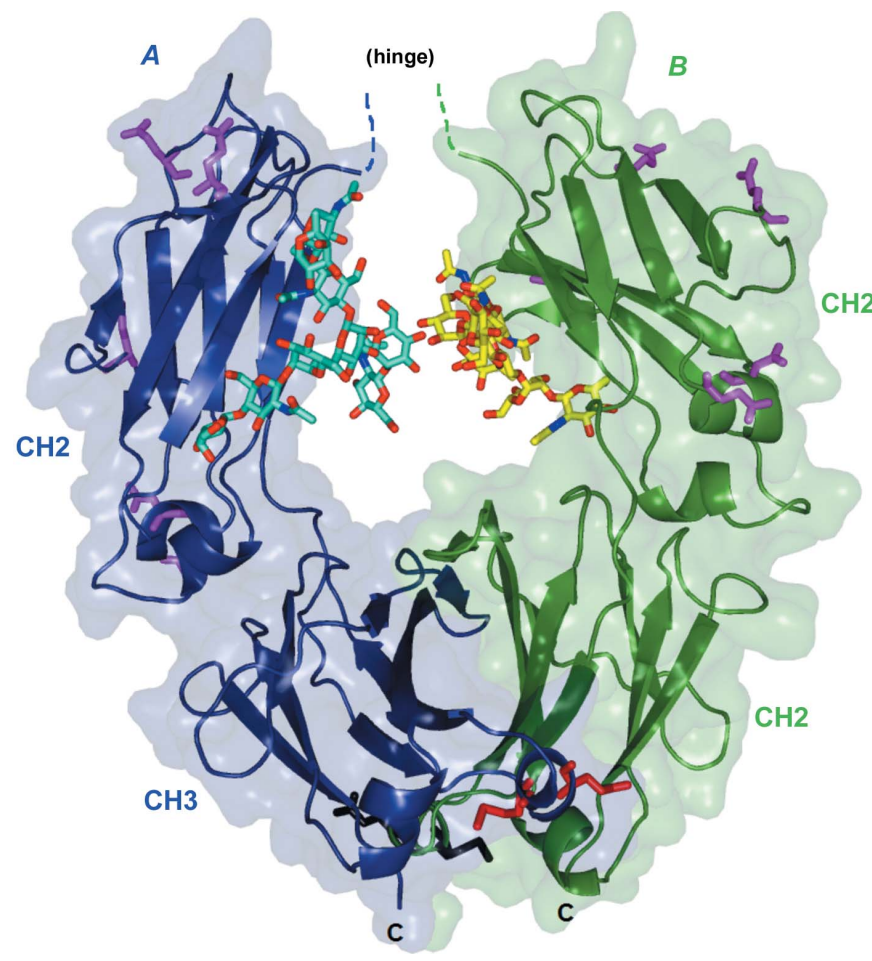

Figure 2

Overall arrangement of the $\mathrm{Fc}$ structure, with subunit $A$ colored blue and subunit $B$ in green. The N-terminal location of the hinge is at the top center and the C-termini are shown at the bottom. Each chain comprises two domains labeled $\mathrm{CH} 2$ and $\mathrm{CH} 3$. Glycans are shown centrally as bright sticks. Each subunit has seven sites of commonly observed allotypic variation, which are also shown as sticks (see §3). All figures were prepared using PyMOL (DeLano, 2002).
The crystal organization builds on the dominant contact shown in Supplementary Fig. S3, which is unique in that it has nearly perfect twofold symmetry despite being between the $A$ and $B$ chains so that it is only pseudosymmetric. The crystal symmetry is pseudo- $C 222_{1}$. If we imagine rotating the $\mathrm{Fc}$ by about $11^{\circ}$ so that its molecular dyad (arrow in Supplementary Fig. S4) aligns with the $z$ axis, so that the contact in this figure becomes symmetric and the contacts in Fig. S5 become identical, then all of the pseudodyads would become dyads and the crystal would become $C 222_{1}$ with a single chain in the asymmetric unit. Presumably the actual packing is energetically preferred so that the molecule tends to crystallize in the observed lower symmetry. However, there are several reported Fc structures with the corresponding C222 symmetry, for example PDB entries 3c2s, 5vh5 and $5 \mathrm{u} 66$.

The Fc dyad is the symmetry axis of the entire antibody, although the extreme flexibility of the hinge region means that the Fabs normally adopt unique and asymmetric conformations. The $\mathrm{CH} 3-\mathrm{CH} 3$ dimer forms the core of the dyad, with its central interface burying $1122 \AA^{2}$ in PDB entry 5vgp. Since the $\mathrm{CH} 2$ domains and glycans do not appear to interact much (across the dyad), the only other tether holding the antibody together is the hinge disulfides. The Fc elbow between the $\mathrm{CH} 2$ and $\mathrm{CH} 3$ domains, spanned by residues $343-345$ and burying about $40 \AA^{2}$, has some flexibility, which enables the $\mathrm{CH} 2$ domains to adopt somewhat varied conformations (Fig. 1). Systematic analysis of this conformation variation may enable a more complete description of the energy landscape of Fc flexibility and the implications for effector binding. The proposed geometric framework applies regardless of

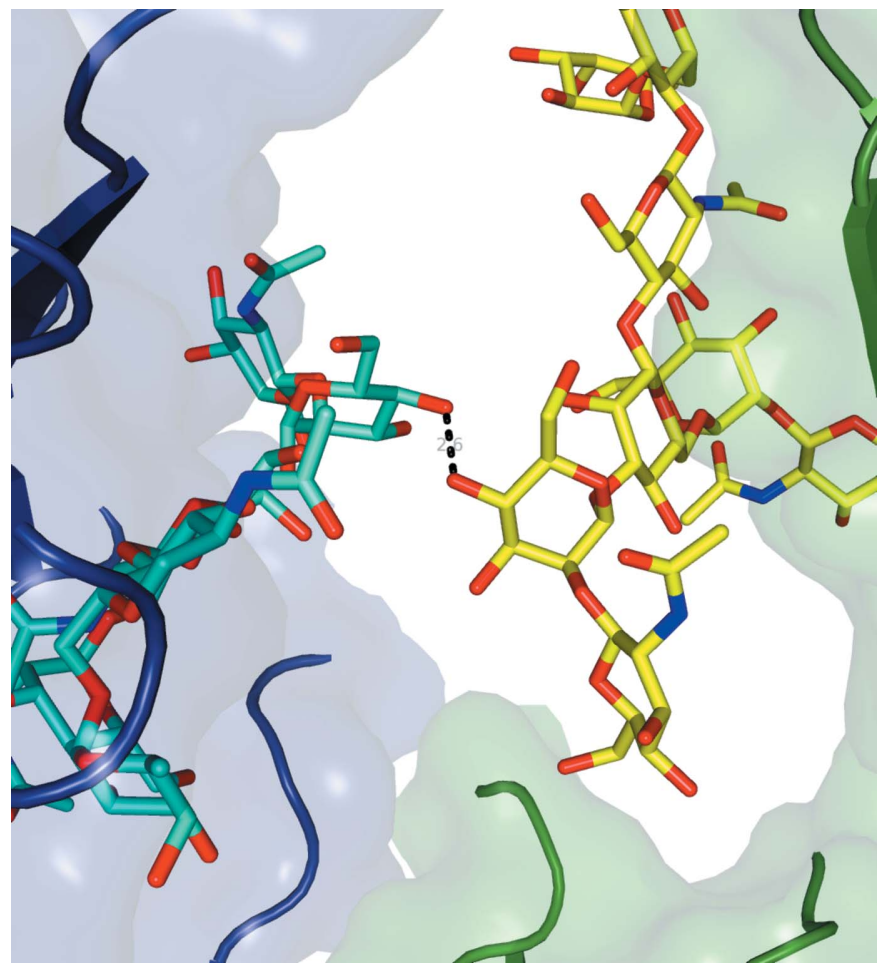

Figure 3

Close-up of the hydrogen bond at the central dyad between the two glycans. 


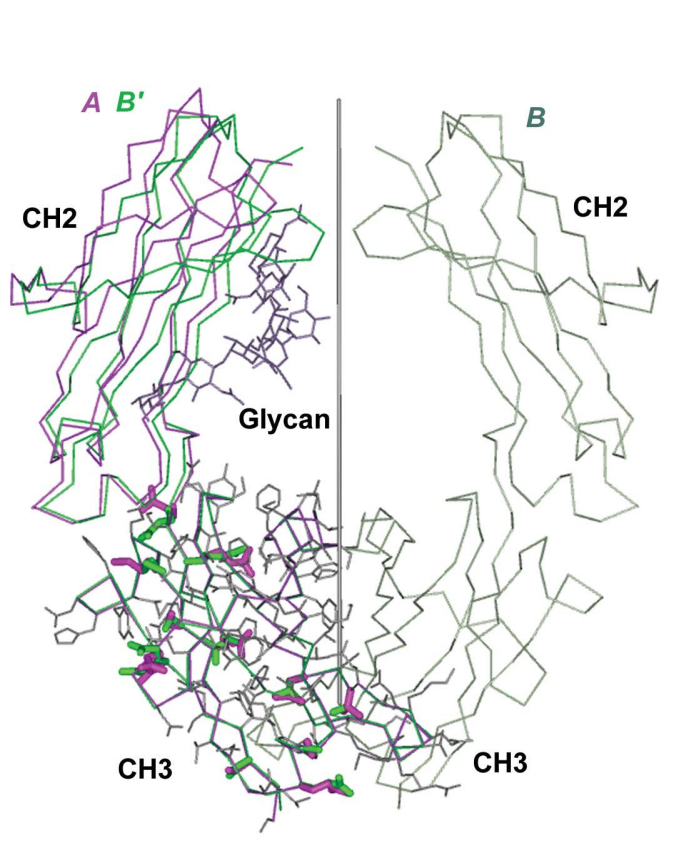

(a)

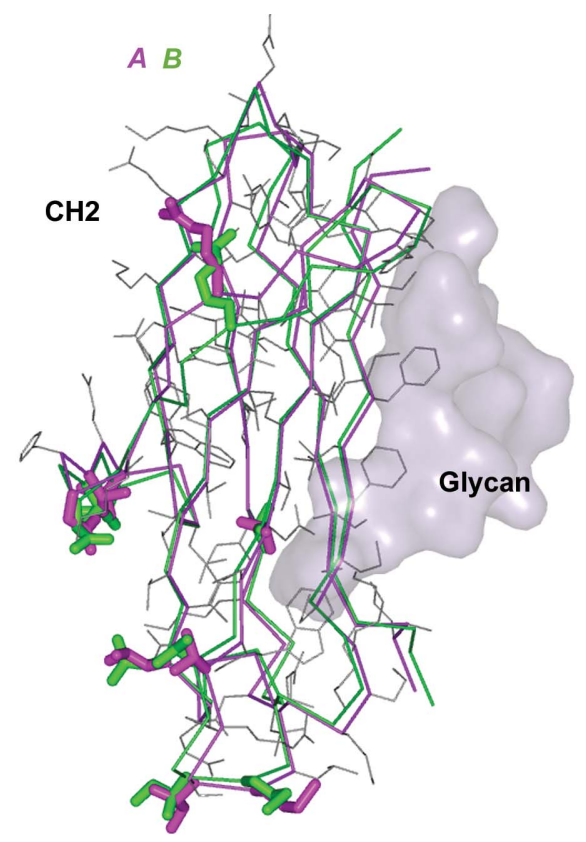

(b)

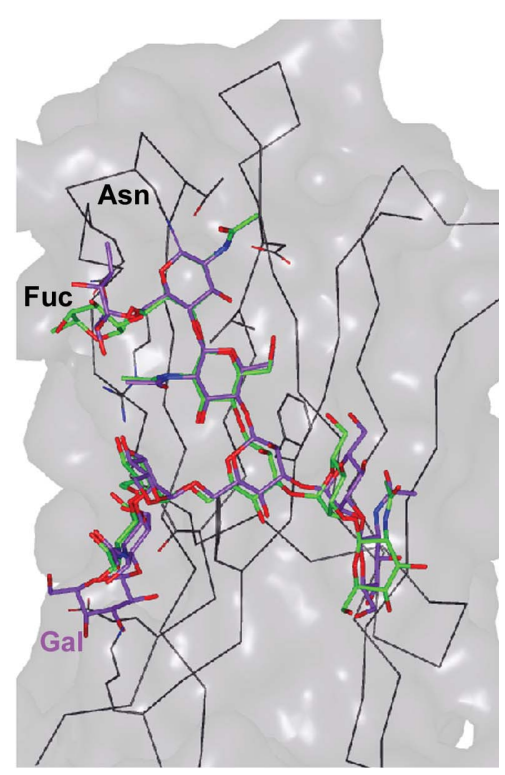

$(c)$

Figure 4

Superpositions showing the similarity of the two subunits in the structure with PDB code 5vgp. In $(a)$ the alignment is based on the CH3 domains, which share a near-perfect dyad (the r.m.s.d. over $103 \mathrm{C}^{\alpha}$ atoms is $0.27 \AA$ ). The $A$ chain is in magenta and the rotated $B$ chain is in green. The $\mathrm{CH} 2$ domains show a misalignment of several angstroms since they do not maintain the $\mathrm{CH} 3$ dyad. The 12 side chains in $\mathrm{CH} 3$ with distinct rotamers in the two subunits (excluding fully solvated charged side chains) are shown as sticks. A single set of thin lines is used for CH3 side chains that agree. The glycan in this picture is for general location only. In $(b)$ the two $\mathrm{CH} 2$ domains are superposed (the r.m.s.d. over $107 \mathrm{C}^{\alpha}$ atoms is $0.95 \AA$ ), showing that the loops (at top and bottom) deviate by about an angstrom. The nine side chains that have different rotamers in the two subunits are shown as sticks. In (c) the two glycans are superposed. The only galactose residue in the structure is at the lower left, where the $A$-chain glycan (magenta) has one additional saccharide unit. The fucose moieties at the upper left show distinct conformations, as do the glucosamines at the lower right.

crystal form; it does not even require a crystal. Since antibody effects follow the binding of monomeric FcR to both subunits of Fc, the intersubunit geometric relationship is likely to be important in signaling. The reference frame enables systematic correlation of Fc modifications (including glycoforms) with variations in the separation of the subunits and other intersubunit shifts that are likely to affect FcR binding.

\section{Conclusion}

The structure of the Fc fragment from the NIST reference antibody RM8671 has been reported at a resolution of $2.1 \AA$ (Fig. 2), along with a brief survey of precedent Fc structures and their conformational variation.

How antigen binding leads to multiple finely regulated effector functions is a central question in immunology. Homeostasis against an ever-changing barrage of microbial and other challenges requires that signaling interactions be information-rich and not simply on/off. The intensity of an immune response is an analog output, a matter of degree, suggesting that $\mathrm{Fc}$ signaling may involve regulation through some form of conformation-dependent code. This appears to apply to the large conformational variation of the hinge, where IgG subtypes have evolved various lengths and flexibilities, and hinge conformation affects the geometry of multivalent pathogen binding. Variation in the Fc elbows is also likely to be a factor in signaling, as it affects the separation of the $\mathrm{CH} 2$ domains, which would in turn affect the accessibility of the glycans to FcR binding.

\section{Acknowledgements}

Certain commercial materials and equipment are identified in order to adequately specify experimental procedures. Such identifications neither imply recommendation or endorsement by the National Institute of Standards and Technology nor do they imply that the material or equipment identified is the best available for the purpose.

\section{Funding information}

IK acknowledges support from National Academy of Sciences National Research Council postdoctoral fellowships. CG acknowledges support from a NIST Summer Undergraduate Research Fellowship. The results shown in this report are derived from work performed at Argonne National Laboratory, Structural Biology Center (SBC) at the Advanced Photon Source. SBC-CAT is operated by UChicago Argonne LLC for the US Department of Energy, Office of Biological and Environmental Research under contract DE-AC02$06 \mathrm{CH} 11357$.

\section{References}

Berman, H. M., Westbrook, J., Feng, Z., Gilliland, G., Bhat, T. N., Weissig, H., Shindyalov, I. N. \& Bourne, P. E. (2000). Nucleic Acids Res. 28, 235-242. 
Caaveiro, J. M. M., Kiyoshi, M. \& Tsumoto, K. (2015). Immunol. Rev. 268, 201-221.

DeLano, W. L. (2002). PyMOL. http://www.pymol.org.

Emsley, P., Brunger, A. T. \& Lütteke, T. (2015). Methods Mol. Biol. 1273, 229-240.

Karageorgos, I., Gallagher, E. S., Galvin, C. V., Gallagher, D. T. \& Hudgens, J. W. (2017). Biologicals, 50, 27-34.

Kennedy, P. J., Oliveira, C., Granja, P. L. \& Sarmento, B. (2018). Crit. Rev. Biotechnol. 38, 394-408.

Krissinel, E. \& Henrick, K. (2007). J. Mol. Biol. 372, 774-797.

Minor, W., Cymborowski, M., Otwinowski, Z. \& Chruszcz, M. (2006). Acta Cryst. D62, 859-866.

Oganesyan, V., Damschroder, M. M., Cook, K. E., Li, Q., Gao, C., Wu,
H. \& Dall'Acqua, W. F. (2014). J. Biol. Chem. 289, 78127824.

Porter, R. R. (1959). Biochem. J. 73, 119-126.

Schiel, J. E., Davis, D. L. \& Borisov, O. V. (2015). Editors. State-of-theArt and Emerging Technologies for Therapeutic Monoclonal Antibody Characterization, Vol. 1. Washington: American Chemical Society.

Schneider, S. \& Zacharias, M. (2012). Mol. Immunol. 51, 66-72.

Teplyakov, A., Zhao, Y., Malia, T. J., Obmolova, G. \& Gilliland, G. L. (2013). Mol. Immunol. 56, 131-139.

Vidarsson, G., Dekkers, G. \& Rispens, T. (2014). Front. Immunol. 5, 520.

Winn, M. D. et al. (2011). Acta Cryst. D67, 235-242. 